\title{
A GESTÃO DA FORMAÇÃO PROFISSIONAL NO CONTEXTO DA GESTÃO DE RECURSOS HUMANOS
}

\author{
Nuno Álvaro Caneca Murchoํㄹ José Eusébio Palma Pacheco² \\ ${ }^{1}$ Doutor em Psicologia - Especialização em Psicologia da Saúde, Responsável do Núcleo de \\ Formação Profissional - Administração Regional de Saúde do Algarve, IP, Faro, Portugal. \\ ${ }^{2}$ Doutor em Psicologia - Especialização em Psicologia da Saúde, Prof. Adjunto Escola Superior \\ de Saúde, Universidade do Algarve, Faro, Portugal. \\ Email para correspondência: nunalvaro@netcabo.pt
}

\begin{abstract}
Resumo
Os elementos mais importantes em qualquer organização são as pessoas. Deste modo, para que as organizações possam ser competitivas e dar resposta às necessidades e aos desafios que se lhes colocam, é fundamental que os seus colaboradores estejam satisfeitos, envolvidos e comprometidos com o seu trabalho. Neste sentido, é importante que as organizações desenvolvam e implementem politicas eficazes de gestão das pessoas, nas quais, a gestão da formação profissional surge com um dos fatores cruciais para as mesmas. Considerando-se então a importância desta temática, com este artigo procurou-se efetuar uma breve reflexão teórica sobre a gestão da formação profissional, a partir de uma revisão narrativa da literatura, com o objetivo de se poder contribuir para um melhor conhecimento da mesma. Assim sendo, concluiu-se que a gestão da formação profissional é um processo complexo, que permite a implementação das políticas de formação profissional das organizações, pelo que pode ser considerada um elemento estruturante ao nível da gestão de recursos humanos nas mesmas, quer a nível individual como organizacional.
\end{abstract}

Palavras-chave: gestão da formação profissional, formação profissional, gestão de recursos humanos.

\begin{abstract}
The most important elements in any organization are the people. Thus, in order for organizations to be competitive and respond to their needs and challenges, it is essential that their employees are satisfied, involved and committed to their work. In this way, it is important that the organizations develop and implement effective people management policies, in which, the management of professional training appears as one of the crucial factors for them. Considering then the importance of this thematic, with this article be sought to carry out a brief theoretical reflection on the management of professional training, based on a narrative review of the literature, for the purpose of to contribute to a better knowledge of it. Therefore, it was concluded that the management of professional training is a complex process, which allows the implementation of the professional training policies of organizations, so it can be considered a structuring element to the level of human
\end{abstract}


resources management in the same organizations, either at the individual level as well as the organizational level.

Keywords: professional formation management, professional management, human resources management.

\section{Introdução}

Os elementos mais importantes em qualquer organização são as pessoas. Deste modo, para que as organizações possam ser competitivas e dar resposta às necessidades e aos desafios que se lhes colocam, num mundo em constante mudança e com exigências por parte dos seus clientes cada vez maiores e mais complexas, é fundamental que os seus colaboradores estejam satisfeitos, envolvidos e comprometidos com o seu trabalho.

Pode-se então dizer, que o desenvolvimento e a implementação de políticas eficazes de gestão de pessoas nas organizações, são fatores essenciais para o sucesso organizacional, sendo crucial a aquisição e aprofundamento de conhecimentos e habilidades que permitam dotar os seus trabalhadores de competências especificas e diferenciadoras que thes permitam otimizar o seu desempenho laboral.

O que levanta preocupações acrescidas com a gestão da formação profissional, aos diferentes níveis, designadamente na determinação da necessidades formativas, no planeamento da formação em termos genéricos e específicos, na implementação e operacionalização do plano de formação e na adesão dos seus destinatários ao mesmo, e finalmente na avaliação das ações de formação realizadas, seja na perspetiva individual (isto é, dos formandos), como na perspetiva organizacional, (ou seja, nos ganhos que representou para a organização), permitindo introduzir alterações e melhorias quer nos planos em curso como nos futuros planos.

Assim, com este artigo pretende-se realizar uma breve reflexão teórica sobre a temática da gestão da formação, a partir da revisão narrativa da literatura consultada, que possa contribuir para um melhor conhecimento desta temática. 


\section{A Formação Profissional: o que é?}

Uma vez que a finalidade deste artigo é refletir sobre a gestão da formação profissional (FP), é importante que em primeiro lugar se defina o elemento principal desta discussão, que é a FP propriamente dita.

$\mathrm{Na}$ verdade, e apesar de ser um termo ou designação comumente utilizada, a todos os níveis, está longe de ter uma definição única, ou mesmo até, de ter uma definição concreta. De qualquer forma, e embora de não haja a pretensão de se encontrar uma definição que seja única ou a definição concreta para este conceito, uma vez que é sobre ele que se vai trabalhar, importa enquadrar-se o mesmo.

Neste sentido, e a partir da revisão da literatura efetuada, considera-se que a FP pode ser entendida em duas dimensões, que se entrecruzam, sendo que uma não exclui a outra, e que são as dimensões individual e organizacional.

No que respeita à dimensão individual, entende-se aqui a FP como o processo global e permanente de aquisição e desenvolvimento de conhecimentos, competências e modificação de atitudes, exigidas para o exercício de uma atividade profissional ou para a melhoria do desempenho, no sentido de melhorarem a eficácia pessoal, de equipa e organizacional e que não confere grau académico (AGUINIS; KRAIGER, 2009; LOURENÇO, 2015; PORTUGAL, 2016).

Numa perspetiva organizacional, é sugerido na literatura consultada que forma como as empresas arquitetam e organizam o conhecimento em sintonia com as suas atividades e no contexto das suas culturas, e como moldam e desenvolvem a eficiência organizacional através do uso das competências dos seus trabalhadores, orientada para a melhoria das operações e dos resultados econômicos das empresas e permitindo às organizações gerir os recursos humanos de forma flexível, atualizá-los e adequá-los às politicas organizacionais (RIBEIRO, 2016; RODRIGUES, 2016; FERNANDES, 2017).

Desta forma, fala-se de formação quando se trabalha com adultos, para distinguir do ensino inicial que se dirige às crianças, aos adolescentes ou aos estudantes, onde, nomeadamente as crianças, são colocadas em situação de objeto do ensino, enquanto que na formação o adulto está na posição de 
sujeito, ao conservar, na medida do possível, a iniciativa e o controlo do seu itinerário de aprendizagem (TOJAL, 2011).

Ou seja, a formação tem o seu enfase no mundo laboral, enquanto a educação no desenvolvimento pessoal do individuo, devendo a primeira ser uma continuação da segunda, como faces da mesma moeda, uma vez que ambas confluem para um denominador comum, que é a aprendizagem dos indivíduos tendo em vista o desenvolvimento de diferentes competências (CARVALHO, 2016; FERNANDES, 2017).

\section{Algumas Facetas da Formação Profissional}

A formação, e neste caso a formação profissional (FP), implica ensinar e aprender, ou seja, aquilo que se designa pelo processo de ensinoaprendizagem, a partir do qual se pretende que o aluno ou neste caso o formando, possa aprender a aprender, o que nas áreas da saúde compreende aprender a conhecer, a fazer, a conviver e a ser (MITRE et al. 2008).

Deste modo, a formação procura provocar um processo de ensinoaprendizagem através de experiências pertinentes tão próximas, quanto possível, das práticas sociais, sendo as suas intenções mais vastas e ambiciosas que as do ensino, propriamente dito, em que mais que a aquisição de um saber, tende para a aprendizagem de um saber fazer, constituído por condutas e práticas, e para o desenvolvimento de um saber estar, relacionado com atitudes e comportamentos que permitem aos sujeitos alargar o seu campo de compreensão e de ação (TOJAL, 2011).

Pode-se então dizer que os processos de aprendizagem são inerentes ao desempenho de qualquer função organizacional, sendo esta aprendizagem uma constante nas organizações, que é mais ou menos formal e integra práticas de gestão permanentes ou apenas ocasionais, embora seja expetável, nomeadamente nas áreas da saúde, que no primeiro contato com a sua função, o trabalhador deva mobilizar saberes, tenham eles já sido previamente adquiridos no sistema de ensino ou em simultâneo com o saber-fazer prático (RODRIGUES, 2016). 
Ao se abordar a formação, e neste caso particular, da FP é comum a utilização de diferentes conceitos, que diferenciam diferentes facetas da mesma.

Deste modo, e a partir da literatura consultada, pode-se dizer que há três grandes facetas da FP, que acompanham o trabalhador desde o inicio do seu ingresso no mercado de trabalho e ao longo da sua vida laboral, que são a FP inicial e a contínua (TOJAL, 2011; RIBEIRO, 2016; RODRIGUES, 2016), e mesmo pessoal, que é a formação ao longo da vida (COSTA, 2015).

A formação inicial é a formação básica essencial para o início de qualquer profissão, designadamente no caso das profissões mais diferenciadas (como é o caso das profissões da saúde), ou seja, é a formação que visa não só a aquisição das capacidades indispensáveis para o exercício de uma determinada função ou profissão, como também habilita para o seu desempenho (RIBEIRO, 2016).

Por outro lado, considera-se que a formação continua é um processo destinado a aperfeiçoar o desenvolvimento profissional do trabalhador, nas suas mais variadas vertentes e dimensões (TOJAL, 2011; CARVALHO, 2016), que decorre em ligação estreita entre a formação formal e a atividade de trabalho, com uma diversidade dos lugares de formação e da sua combinação (e.g., formação na empresa, postos de trabalho, instituições de formação) e de formadores (e.g., profissionais, fornecedores de equipamento, da empresa) (RODRIGUES, 2016).

Já a formação ao longo da vida, é entendida como toda e qualquer aprendizagem que as pessoas adquirem ao longo de toda a sua vida seja em contextos formais, não formais ou informais, sendo uma tarefa contínua da sociedade e do indivíduo, que se estende a todas as áreas da vida, desde que nasce até que morre (COSTA, 2015).

Outras facetas da FP, mais específicas embora interligadas com estes três últimos conceitos, são entre outras, a formação em serviço, a reciclagem, a reconversão profissional, a formação tutorial, a formação pela atividade do trabalho e a formação através da organização qualificante (TOJAL, 2011; BASTOS, 2014; RIBEIRO, 2016; RODRIGUES, 2016). 
A este respeito, de referir que é exequível a coexistência de diferentes modalidades de formação e que a mesma modalidade de formação pode até ser utilizada com objetivos diferentes (RODRIGUES, 2016).

Fazendo uma descrição sucinta destes conceitos, pode-se dizer que a formação em serviço visa colmatar as necessidades especificas de formação dos elementos de uma equipa/serviço, no local de trabalho (TOJAL, 2011; BASTOS, 2014); a reciclagem está vocacionada/direcionada para a atualização de conhecimentos; a reconversão profissional possibilita a adaptação a uma nova atividade profissional, através da aquisição de uma qualificação diferente da que o trabalhador detinha (RIBEIRO, 2016); a formação tutorial consiste no apoio, acompanhamento e integração dos trabalhadores juniores pelos trabalhadores séniores; a formação pela atividade de trabalho é o processo autoformativo que decorre do exercício de uma determinada função ou profissão, nomeadamente através do processo de resolução dos problemas que se colocam nos diferentes postos e contextos de trabalho; e a formação através da organização qualificante, resulta do próprio meio organizacional enquanto gerador de competências por si, através das redes de circulação de informação e dos procedimentos de resolução coletiva de problemas (RODRIGUES, 2016).

\section{$4 \quad$ A Gestão do Conhecimento}

Atualmente o conhecimento tornou-se um recurso chave para as organizações, e é um elemento básico do capital humano e das competências individuais (através do desenvolvimento de atitudes e habilidades adequadas), assentando o sistema de gestão do conhecimento nas pessoas, entre outros aspetos, porque são elas que realizam os processos ligados à identificação, criação, obtenção, purificação, proteção e transferência do conhecimento (LENDZION, 2015).

Na perspectiva das organizações, e de um modo geral, reconhece-se o conhecimento como um recurso de fundamental importância em qualquer setor de atividade, considerando que é um fator essencial para o desenvolvimento e crescimento organizacional, seja em organizações do setor privado, publico ou social (JANNUZZI; FALSARELLA; SUGAHARA, 2016). 
Por outro lado, o conhecimento, que é o processo e o resultado da relação entre $\mathrm{o}$ indivíduo que conhece e o objeto a ser conhecido ou já conhecido, aumenta quando partilhado, e pode ser utilizado pelas organizações como um elemento de inovação, gerador de mudanças de desenvolvimento ou radicais ou como matéria-prima geradora de bens e serviços, sendo para algumas, um recurso estratégico que permite processar a transformação de conhecimentos sensíveis, individuais e mutáveis em ativos do conhecimento, que por serem intangíveis ao invés de perderem valor com o uso, aumentamno (FREIRE; SPANHOL, 2014).

Assim, a gestão do conhecimento (GC) pode ser entendida como um um processo de criação e utilização do conhecimento que permite melhorar a efetividade das atividades das organizações, através da coordenação deliberada e sistemática das pessoas, de tecnologia, de processos e da estrutura de uma organização, e da organização do fluxo de conhecimento entre a criação e a aplicação do conhecimento, cujas finalidades são a agregação de valor através da reutilização do conhecimento e da inovação, e a promoção da aprendizagem organizacional continua (LENDZION, 2015; JANNUZZI; FALSARELLA; SUGAHARA, 2016).

A este respeito ainda, e para que o processo de GC seja eficaz, as suas três dimensões devem repetir-se ciclicamente nas organizações, sendo estas dimensões as seguintes: criação e retenção do conhecimento a aplicar para alcançar os objetivos organizacionais; compartilha, conversão, organização e disseminação do conhecimento entre as pessoas nas organizações; e aplicação do conhecimento para a criação de valor para a organização e para os seus clientes (FERRARESI et al, 2014).

\section{A Gestão da Formação}

A gestão da formação (GF) entende-se como um processo de análise de situações e recursos, de gestão de redes relacionais, de tomada ou proposta de decisões de politica e de realizações formativas, permitindo a otimização dos recursos atribuídos, de planeamento e realização de ações de formação profissional, avaliação e reformulação dos resultados obtidos e apresentação e discussão dos mesmos com os decisores, bem como de relação continua e 
retroativa com os seus clientes, visando a tomada de decisões no que respeita a todas as fases intrinsecamente ligadas à formação e aos seus participantes, em articulação permanente com a gestão de recursos humanos $(R H)$ e os próprios $\mathrm{RH}$ da organização onde se insere, contribuindo por essa via para a melhoria da eficiência, eficácia, desempenho e produtividade da organização com a qual os seus destinatários mantém uma relação laboral (FERNANDES, 2017).

De qualquer forma, é importante mencionar que a GF, assenta obrigatoriamente na política de formação da própria organização, sendo esta um dos pontos essenciais do processo de gestão e desenvolvimento de $\mathrm{RH}$ na mesma (FERNANDES, 2017).

Até porque, é sugerido na literatura consultada que quanto mais eficaz é a gestão dos $\mathrm{RH}$ numa determinada organização, maior é a possibilidade de sucesso, estando este resultado intrinsecamente relacionado com as condições de trabalho que se oferecem aos trabalhadores, com a oportunidade de progresso através da formação formal e informal, e com a criação de um ambiente laboral propicio à criatividade (BASTOS, 2014).

Assim sendo, pode-se dizer que a GF decorre das políticas e das estratégias determinadas pelas administrações das organizações, a que respeitam, nomeadamente no que concerne à gestão de $\mathrm{RH}$, até porque é esse o core da formação profissional: as pessoas das organizações, independentemente do seu nível de decisão, sejam eles trabalhadores de base, chefias, diretores ou administradores de topo, incluindo inclusive, os próprios empresários, no caso das organizações privadas.

Deste modo, a GF deve adaptar-se às realidades de cada organização e às efetivas possibilidades de intervenção formativa, pressupondo este processo uma capacidade de pensar em missões e não em tarefas, de comando do processo e colocação sob controlo de sistemas em vez de gestão meramente administrativa, que permitam levar a formação à prática, seja através das fases do ciclo de gestão da formação, dos diagnósticos à avaliação da formação, ou ainda das práticas de formação propriamente ditas, mediante situações de formação formal, informal e não formal (OLIVEIRA, 2017). 
Para tal, assenta em cinco pilares, que a literatura designa como os cinco pilares da GF, e que são os seguintes: a adequação dos serviços prestados face às necessidades de formação existentes; o processo de decisão por detrás de cada formação, tendo por base as políticas da organização, bem como o plano de formação existente nesta; o processo de compra ou a produção de programas de formação; o acompanhamento e controlo da formação; e a visibilidade dos resultados nos postos de trabalho de cada trabalhador, de modo a que haja maior produtividade e rentabilidade de conhecimentos e competências no seio da organização (FERNANDES, 2017).

Considerando a literatura consultada, pode-se dizer que a GF engloba várias funções ou fases, as quais podem ser organizadas sequencialmente da seguinte forma: o estabelecimento da orientação geral, desde o pré-diagnóstico até à explicitação da política da formação, e que visa determinar a orientação geral da ação formativa, correspondendo à politica de formação; o levantamento e análise das necessidades de formação, que é determinado pela política de formação a que se dá sequência; a formulação dos objetivos, a partir do levantamento e análise de necessidades e da politica de formação; a determinação do conteúdo da formação, em função do levantamento e análise das necessidades formativas e dos objetivos formulados, mas também dos seus destinatários (formandos); a elaboração da proposta de ação formativa (o plano de formação) para ser submetida a análise e aprovação da hierarquia competente; o desenvolvimento da ação formativa, que diz respeito à preparação e coordenação das ações, incluindo a definição dos programas formativos, bem como a escolha de quem vai participar nas ações (formadores e destinatários), dos espaços e dos meios a utilizar, e eventualmente a elaboração de cadernos de encargos formativos e compra de serviços de formação; e finalmente, o controlo da ação formativa, ou seja a avaliação, que permite obter feedback sobre a ação, levando ou não a um reajustamento do plano, e mesmo até a um novo levantamento de necessidades de formação (CARDIM, 2012; RODRIGUES, 2016; FERNANDES, 2017).

Por outro lado, a GF deve ser coordenada por um elemento, que se pode designar como o gestor de formação, e que tem como função a gestão de todo o processo de formação profissional, desde o estabelecimento da orientação 
geral, até ao controlo da ação formativa, articulando para tal com todos os elementos da organização, não tanto como um especialista, mas principalmente como um par, com capacidade para comunicar, mas também para se adaptar à mudanças de contexto e de ambiente externo e interno da organização e das suas necessidades, em concordância com a estratégia da organização (FERNANDES, 2017).

\section{Conclusão}

A partir da literatura consultada, verificamos que a formação profissional (FP) é um processo de ensino-aprendizagem, que é crucial para as organizações e para os seus elementos mais importantes, que são as pessoas que nelas trabalham, e que permite, através de experiências tão próximas quanto possível, das práticas sociais (MITRE et al. 2008), principalmente ao nível das formações inicial e contínua (TOJAL, 2011; RIBEIRO, 2016; RODRIGUES, 2016), atingir as seguintes finalidades:

- Ao nível individual, a aquisição e desenvolvimento de conhecimentos, competências e modificação de atitudes, exigidas para o exercício de uma atividade profissional ou para a melhoria do desempenho (AGUINIS; KRAIGER, 2009; LOURENÇO, 2015; PORTUGAL, 2016), e;

- Ao nível organizacional, a arquitetura e organização do conhecimento em sintonia com as suas atividades e no contexto das suas culturas, e como moldam e desenvolvem a eficiência organizacional através do uso das competências dos seus trabalhadores (RIBEIRO, 2016; RODRIGUES, 2016; FERNANDES, 2017).

Por outro lado, o conhecimento, que é um recurso chave para as organizações, e que, quer as pessoas como as organizações, vão adquirindo, desenvolvendo e organizando, necessita de ser gerido, em termos individuais (Lendzion, 2015) ou organizacionais (JANNUZZI; FALSARELLA; SUGAHARA, 2016).

Este processo de criação e utilização do conhecimento, é aqui entendido como um recurso estratégico que permite processar a transformação de conhecimentos sensíveis, individuais e mutáveis em ativos do conhecimento, que por serem intangíveis ao invés de perderem valor com o uso, aumentam- 
no (FREIRE; SPANHOL, 2014), promovendo a agregação de valor através da reutilização do conhecimento e da inovação, e a promoção da aprendizagem organizacional continua, designada por gestão do conhecimento (LENDZION, 2015; JANNUZZI; FALSARELLA; SUGAHARA, 2016), e que está intrinsecamente ligada à $\mathrm{FP}$, da qual é indissociável, até porque a FP promove a criação e desenvolvimento de conhecimento nas organizações.

Deste modo, a gestão da formação é um processo de análise de situações e recursos, de gestão de redes relacionais, de tomada ou proposta de decisões de política e de realizações formativas, que assenta obrigatoriamente na política de formação da própria organização, sendo um elemento estruturante do processo de gestão e desenvolvimento dos seus recursos humanos, que engloba sequencialmente o estabelecimento da orientação geral, o levantamento e análise das necessidades de formação, a formulação dos objetivos, a determinação do conteúdo da formação, a elaboração da proposta de ação formativa (plano de formação), o desenvolvimento da ação formativa, e finalmente, o controlo da ação formativa (CARDIM, 2012; RODRIGUES, 2016; FERNANDES, 2017).

\section{Referências}

AGUINIS, Herman; KRAIGER, Kurt. Benefits of Training and Development for Individuals and Teams, Organizations, and Society. Annual Review of Psychology, Palo Alto (CA - USA), v. 60, p. 451-74, 2009. Disponível em: $<$ https://doi.org/10.1146/annurev.psych.60.110707.163505>. Acesso em: 6 set. 2018.

BASTOS, Ana Luísa P. Gonçalves. O enfermeiro gestor na formação em serviço - a família em contexto hospitalar. 2014. 141 p. Dissertação (mestrado em direção e chefia de serviços de enfermagem) - Escola Superior de Enfermagem do Porto, Porto, PT. Disponível em: <http://hdl.handle.net/10400.26/9507>. Acesso em: 2 set. 2018.

CARDIM, José Casqueiro. A gestão da formação nas organizações - a formação na prática e na estratégia das organizações. 2. ${ }^{a}$ ed.. Lisboa, PT: Lidel - Edições Técnicas Lda, 2012. 176 p.

CARVALHO, Eliza M. Arezes de Castro. Do diagnóstico de necessidades de formação à conceção de uma proposta de plano de formação. 2016. 254 p. 
Relatório de estágio (mestrado em educação). Instituto de Educação, Universidade do Minho, Braga, PT. Disponível em: <http://hdl.handle.net/1822/45243>. Acesso em: 24 jul. 2018.

COSTA, Etelberto. Aprendizagem ao longo da vida: conceito e políticas, Dirigir \& Formar, Lisboa (PT), n. 11, p. 52-57, abr./jun. 2015. Disponível em: $<$ https://www.iefp.pt/documents/10181/702841/D\%26F+n.\%C2\%BA\%2011.pdf/be3a67 e2-2f81-4a93-8d13-150a6552661e>. Acesso em: 30 jul. 2018.

FERNANDES, Liliana Pereira. Formação, Gestão e Avaliação da Formação: Conceções e Práticas num Grupo Empresarial. 2017. 217 p. Relatório de estágio (mestrado em educação) - Instituto de Educação, Universidade do Minho, Braga, PT. Disponível em: < http://hdl.handle.net/1822/54842>. Acesso em: 24 jul. 2018.

FERRARESI, Alex Antonio et al. Os impactos da gestão do conhecimento na orientação estratégica, na inovatividade e nos resultados organizacionais: uma survey com empresas instaladas no Brasil, Revista de Administração Mackenzie, São Paulo, SP, v. 15, n. 2, p. 199-231, mar./abr. 2014. Disponível em: <http://www.scielo.br/pdf/ram/v15n2/v15n2a08.pdf >. Acesso em: 25 jul. 2018.

FREIRE, Patricia de Sá; SPANHOL, Fernando José. Conhecimento organizacional: produto ou processo? Perspectivas em Gestão \& Conhecimento, João Pessoa, PB, v. $4, \quad$ n. $1, \quad$ p. 3-21, jan./jun. 2014. Disponível em: <http://periodicos.ufpb.br/ojs2/index.php/pgc >. Acesso em: 15 set. 2018.

JANNUZZI, Celeste S. Corrêa; FALSARELLA, Orandi M.; SUGAHARA, Cibele R. Gestão do conhecimento: um estudo de modelos e sua relação com a inovação nas organizações, Perspectivas em Ciência da Informação, Belo Horizonte, MG, v. 21, n. 1, p. 97-118, jan./mar. 2016. Disponível em: <http://dx.doi.org/10.1590/19815344/2462>. Acesso em: 25 jul. 2018.

LENDZION, Jaroslaw Piotr. Human resources management in the system of organizational knowledge management. Procedia Manufacturing, Lodz, PL, v. 3, p. 674-680, 2015. Disponível em: <https://doi.org/10.1016/j.promfg.2015.07.303>. Acesso em: 24 jul. 2018. 
LOURENÇO, Teresa M. S. Pinto. A Importância da Formação Profissional enquanto Investimento em Capital Humano. 2015. 59 p. Relatório de estágio (mestrado em economia). Faculdade de Economia, Universidade de Coimbra, Coimbra, PT. Disponível em: <http://hdl.handle.net/10316/29695>. Acesso em: 3 set. 2018.

MITRE, Sandra Minardi et al.. Metodologias ativas de ensino-aprendizagem na formação profissional em saúde: debates atuais. Ciência \& Saúde Coletiva, Rio de Janeiro, RJ, v. 13, Suppl. 2, 2133-2144, 2008. Disponível em: <https://dx.doi.org/10.1590/S1413-81232008000900018>. Acesso em: 10 set. 2018.

OLIVEIRA, Tiago A. Gomes. Políticas e práticas de formação das unidades de saúde hospitalares: um estudo multicasos dos centros de formação dos hospitais. 2017. 114 p. Dissertação (mestrado em educação). Instituto de Educação, Universidade do Minho, Braga, PT. Disponível em: <http://hdl.handle.net/1822/54700>. Acesso em: 26 set. 2018.

PORTUGAL. Decreto-Lei n.ำ 86-A/2016, de 29 de dezembro. Diário [da] República, Lisboa, PT, 29 dez. 2016. Série I, 3o Suplemento, n. 249. Disponível em: <https://dre.pt/application/file/a/105658997>. Acesso em: 10 jul. 2018.

RIBEIRO, Joana F. Santos. Práticas da Formação, Clima e Cultura Organizacional: Estudo de caso numa organização empresarial. 2016. 165 p. Relatório de estágio (mestrado em educação). Instituto de Educação, Universidade do Minho, Braga, PT. Disponível em: <http://hdl.handle.net/1822/45303>. Acesso em: 24 jul. 2018.

RODRIGUES, Magda Dias. Desenvolvimento e atualização de competências: a formação como ferramenta de gestão estratégica de recursos humanos - Estudo de Caso. Dissertação (mestrado em marketing e comunicação). 2016. 131 p. Escola Superior de Educação, Instituto Politécnico de Coimbra, Coimbra, PT. Disponível em: <http://hdl.handle.net/10400.26/17699 >. Acesso em: 25 jul. 2018.

TOJAL, Ana M. A. Fernandes. A perceção dos enfermeiros sobre a formação em serviço. 2011. 159 p. Dissertação (mestrado em enfermagem médico-cirúrgica). Escola Superior de Enfermagem de Coimbra, Coimbra:. Disponível em: <http://repositorio.esenfc.pt/?url=b6elM16b $>$. Acesso em: 2 set. 2018. 